\title{
Thermoacoustic Effects in High-Speed Compressible Transitional and Turbulent Boundary Layers
}

\author{
Konstantinos Ritos ${ }^{1}$, Ioannis W. Kokkinakis ${ }^{2}$, Dimitris Drikakis ${ }^{3}$ \\ University of Strathclyde, Glasgow, G1 $1 X W, U K$
}

S. Michael Spottswood ${ }^{4}$

Air Force Research Laboratory, Wright Patterson AFB, OH 45433-7402, USA

\begin{abstract}
A numerical investigation of the thermal and acoustic effects in high-speed compressible flows is presented. Two case studies are considered: i) transition to turbulence in supersonic flows over a flat plate, and ii) supersonic shock wave turbulent boundary layer interaction (SWTBLI) over a compression ramp. Implicit Large Eddy Simulations (iLES) are performed using the second and fifth order Monotone-Upstream Central Scheme for Conservation Laws (MUSCL) and the ninth order Weighted Essentially Non-Oscillatory (WENO) schemes. The aim of this study is twofold: i) to examine the acoustic and thermal effects associated with transitional and turbulent boundary layers, particularly in the near wall region; ii) to investigate the effects of numerical accuracy on acoustic and thermal loading. The results are compared with theoretical models, Direct Numerical Simulations (DNS) and experiments.
\end{abstract}

\footnotetext{
${ }^{1}$ Lecturer, University of Strathclyde, Glasgow, UK, Member of AIAA

Corresponding author: konstantinos.ritos@strath.ac.uk.

${ }^{2}$ Lecturer, University of Strathclyde, Glasgow, UK.

${ }^{3}$ Professor of Engineering Science \& Executive Dean of the Faculty of Engineering at the University of Strathclyde, Glasgow, UK, Senior Life Member AIAA.

${ }^{4}$ Senior Aerospace Engineer, Structural Sciences Center, Wright Patterson AFB, USA
} 


\section{Introduction}

Understanding of thermoacoustic loads in high-speed compressible flows will become increasingly important in the design of future supersonic and hypersonic planes because they can affect the integrity of the structure. Turbulent boundary layers (TBL) that exist in highspeed flows impose random pressure acoustic loads on structural elements. These loads are the source of acoustic fatigue, a critical issue that all high-speed flying structures face. Developing a tool that can make more accurate predictions about the positions and magnitudes of pressure fluctuations along with the position of high thermal loads will enable engineers to push designs closer to the edge of the performance envelope, with lower safety factors and possibly more optimal designs.

Experiments and numerical simulations can work together to broaden the range of flow conditions to be investigated. Several recent publications have highlighted the capabilities of direct numerical simulation (DNS) for pressure fluctuations beneath a TBL $[1,2,3]$. Ostoich et al. [3] included the behavior of a compliant panel and showed how this can fundamentally change the turbulent statistics of the TBL. Note that simulations of supersonic/hypersonic TBLs under realistic flight conditions have not yet been performed.

Even though numerical methods based on DNS offer the necessary high fidelity, they impose strict spatial resolution constraints that lead to a very high computational cost. Because they have less strict grid requirements, classical large eddy simulation (LES) methods are more efficient. However, they require the use of a low-pass filtering operation that produces sub-grid scale terms requiring additional modeling, which in turn introduces further numerical errors. In an effort to find a compromise between accuracy and computational cost, the concept of implicit LES (iLES) emerged from observations reported by Boris et al. [4]. This method has been successfully applied to model several complex flows in engineering and other fields. Fureby and Grinstein [5] justified the use of iLES in free and wall-bounded flows, while Margolin et al. [6] presented a validation of the method via a theoretical analysis. More recently, two independent publications applied iLES in two different cases, with both concluding that iLES can achieve near DNS results while using significantly less computational resources. In the first of these studies, Kokkinakis and Drikakis [7] presented iLES results of a weakly compressible turbulent channel flow. In the second, Poggie et al. [8] applied iLES to study TBL flows using a simulation setup similar to the one we will present in this paper. This non-exhaustive list of examples shows the effectiveness of iLES in various applications and illustrates that this method has been validated and documented by various authors.

The main constraints of any numerical simulation are its accuracy and computational cost. The commonly used low-order (up to second-order) numerical schemes are highly dissipative and, as a result, produce incorrect predictions even for simple flow geometries such as a flat plate. High-order (HO) schemes in combination with large eddy simulations provide significantly better results. Nonetheless, pressure fluctuations can still be underpredicted, or overpredicted due to numerical dispersion. In recent years, significant progress has been made with regard to the application of high-resolution and HO methods in compressible flows featuring acoustic excitation, supersonic shock wave turbulent boundary layer interaction (SWTBLI), and low-Mach number effects.

Here, we apply numerical schemes up to ninth-order accurate to calculate supersonic transitional flow over a flat plate and supersonic SWTBLI over a compression ramp. Various $\mathrm{HO}$ methods are investigated within the framework of iLES. It is shown that increasing the order of the method leads to a corresponding increase in the accuracy of the iLES results. For the highest-order method investigated, the iLES results offer levels of accuracy equivalent to those of DNS results. The numerical methods are briefly presented in Section II and the results from both cases are presented in Section III. 


\section{Numerical methods}

The in-house block-structured grid code, CNS3D, solves the full Navier-Stokes equations using a finite volume Godunov-type method for the convective terms. The inter-cell numerical fluxes of the convective terms are calculated by solving the Riemann problem using the reconstructed values of the conservative variables at the cell interfaces. The reconstruction stencil is a one-dimensional swept unidirectional stencil. The Riemann problem is solved using the so-called "Harten, Lax, van Leer, and (the missing) Contact" (HLLC) approximate Riemann solver [9]. Two different flux limiting approaches have been implemented in conjunction with the HLLC solver, namely (i) Monotone Upstream-centered Schemes for Conservation Laws (MUSCL) and (ii) Weighted-Essentially-Non-Oscillatory (WENO). In particular, the following schemes are examined:

- MUSCL piecewise linear second-order Monotonized Central (M2) limiter [10];

- MUSCL fifth-order (M5) limiters [11]; and

- WENO ninth-order (W9) schemes [12].

In the low subsonic region of transitional/turbulent boundary layers the accuracy of the above schemes, as well as that of any other scheme, can be further improved by implementing low-Mach corrections (henceforth labeled LM) [13]. This essentially involves an additional numerical reconstruction step of the velocity vector via a progressive central differencing of the velocity components. LM corrections ensure a balanced distribution of the dissipation of kinetic energy in the limit of zero Mach number; therefore, extending the validity of compressible flow codes to Mach numbers as low as $10^{-5}$ is required, particularly required for schemes providing an accuracy less than fifth-order [7]. The viscous terms are discretized by a second-order central scheme. The solution is advanced in time using a fivestage (fourth-order accurate) optimal strong-stability-preserving Runge-Kutta method [14]. Further details of the numerical aspects of the code are given in Refs. [7, 15] and references therein.

\section{Results}

\section{A. Transition to turbulence in supersonic flow over a flat plate}

For the first case, we used a simulation setup similar to the DNS study of Poggie et al. [8]. We considered a Mach 2.25 supersonic flow over a flat plate that features transition to turbulence. The incoming flow had a Reynolds number of $1.5 \times 10^{6}$ based on the freestream properties and the length of the plate $(L)$. Further flow parameters are given in Table 1, while Figure 1 shows a schematic representation of the simulation domain and the boundary conditions.

Table 1: Simulation parameters for the first case.

\begin{tabular}{ccccc}
\hline $\mathrm{L}$ & $\mathrm{U}_{\infty}$ & $\mathrm{P}_{\infty}$ & $\mathrm{T}_{\infty}$ & $\mathrm{M}$ \\
$0.061 \mathrm{~m}$ & $588 \mathrm{~m} / \mathrm{s}$ & $23.8 \mathrm{kPa}$ & $170 \mathrm{~K}$ & 2.25 \\
\hline \hline$\mu_{\infty}$ & $\rho_{\infty}$ & $\mathrm{T}_{\mathrm{w}} / \mathrm{T}_{\infty}$ & $\mathrm{I}$ & $\mathrm{Re}_{\mathrm{L}}$ \\
$1.167 \times 10^{-5} \mathrm{~Pa} \mathrm{~s}$ & $0.488 \mathrm{~kg} / \mathrm{m}^{3}$ & 1.9 & $0.03 \mathrm{U}_{\infty}$ & $1.5 \times 10^{6}$ \\
\hline
\end{tabular}




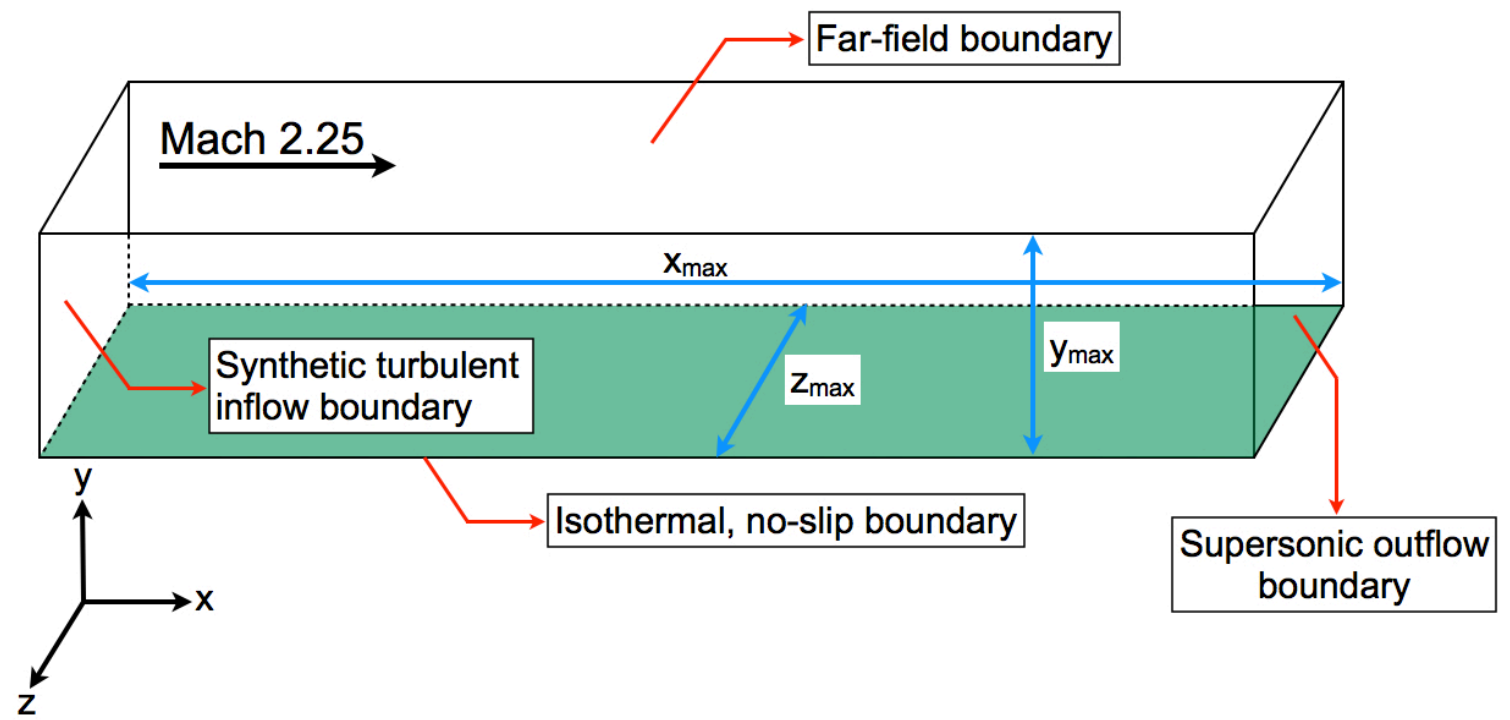

Figure 1: Schematic representation of the simulation domain, highlighting the boundary conditions used.

A synthetic turbulent inflow boundary condition was used to produce a freestream flow with a superimposed random turbulence with an intensity of $\pm 3 \%$ of the freestream velocity. This small perturbation is found sufficient to trigger a bypass transition and turbulence at a downstream position. The spectrum of the introduced turbulence contains energy modes within the Kolmogorov inertial range $(-5 / 3)$; however, large-scale energy modes are absent. In addition, a cutoff at a maximum frequency of $50 \mathrm{MHz}$ was applied becasue the finest mesh used in this study under-resolves higher values.

The mesh resolution effects were investigated using four different meshes (see Table 2). The $\mathrm{y}^{+}$distance was close to 2 , which is sufficient according to previous investigations [7], at least for the highest W9 scheme. The second and fifth order MUSCL schemes have been used in conjunction with the finest mesh only.

Table 2: Mesh parameters for the first case.

\begin{tabular}{ccccc}
\hline & Very fine & Fine & Medium & Coarse \\
\hline $\mathrm{x}_{\max }$ & 1 & 1 & 1 & 1 \\
$\mathrm{y}_{\max }$ & 0.05 & 0.05 & 0.05 & 0.05 \\
$\mathrm{z}_{\max }$ & 0.05 & 0.05 & 0.05 & 0.05 \\
$\mathrm{nx}$ & 2591 & 1727 & 1151 & 864 \\
$\mathrm{ny}$ & 277 & 227 & 151 & 114 \\
$\mathrm{nz}$ & 139 & 92 & 61 & 47 \\
$\Delta \mathrm{x}^{+}$ & 33.24 & 49.88 & 74.87 & 99.76 \\
$\Delta \mathrm{y}^{+}$ & 1.82 & 2.23 & 3.37 & 4.48 \\
$\Delta \mathrm{z}^{+}$ & 31.19 & 47.31 & 71.75 & 93.58 \\
\hline
\end{tabular}

Due to the lack of a friction law describing transitional flows, we calculated the skin friction coefficient and compared it with models of laminar and turbulent flows (Figure 2a). In the initial laminar region of the boundary layer, our calculations show excellent agreement 
with Blasius' friction law. Furthermore, all methods captured the transition effect and the onset of turbulence, showing good agreement with the models of White and van Driest [16, 17]. However, the transition point is delayed for coarser meshes and low-order methods. In addition, M2 predicts a larger transition zone compared to M5 and W9. Note that W9 on a coarse grid can obtain similar accuracy to M2 on a much finer grid, therefore increasing the efficiency of the simulations.

a)

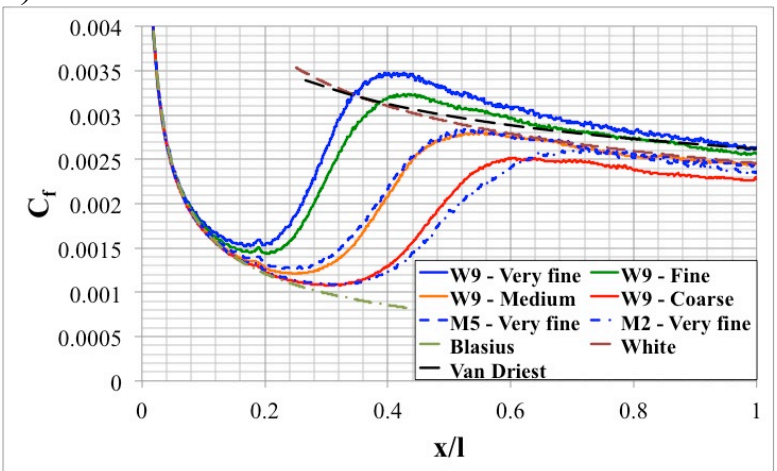

b)

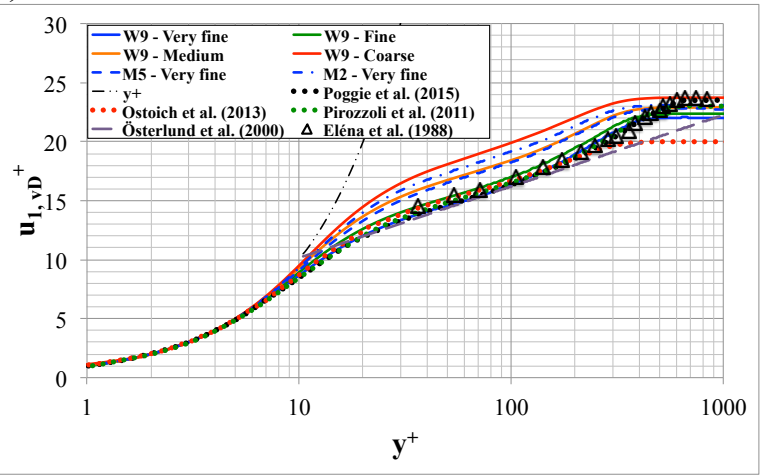

Figure 2: a) Skin friction coefficient $C_{f}$ along the plate. Theoretical models for fully laminar and fully turbulent flows are also presented. b) van Driest transformed streamwise velocity profile as a function of $y^{+}$at the end of the simulation domain. Theoretical, numerical and experimental results from previous publications are also presented.

It is well established that the turbulent boundary layer has a velocity profile that can be described, at least near the wall, by a one-parameter family of profiles, i.e., the logarithmic velocity distribution due to von Karman. This "law of the wall" is expressed as

$$
u_{1, v D}^{+}=\frac{1}{k} \ln \left(y^{+}\right)+C,
$$

where $k$ and $C$ are constants that have to be derived experimentally; and $u_{1, v D}^{+}$is the van Driest transformed velocity. Figure $2 \mathbf{b}$ shows the time-averaged van Driest transformed velocity profiles for all the simulated cases at the end of the plate, where the flow is fully turbulent. In the same figure, we compare our calculations with the DNS results and experimental data at similar Mach numbers. The results of the highest-order scheme, i.e., W9 perfectly collapse to previous DNS [3, 8, 18], experimental [19], and incompressible theoretical [20] results. Note that differences in the results in the above studies are also due to the different Reynolds numbers employed at the probe locations in the simulations and experiments [3]. For example, past DNS studies of fully turbulent flows have been performed at $\operatorname{Re}_{\delta 2}=2000$ [8] and $\mathrm{Re}_{\delta 2}=1552$ [18], while past experiments have been performed at $\mathrm{Re}_{\delta 2}$ $=2600$ [19]. The present results corresponding to the W9 simulations coincide with $\mathrm{Re}_{\delta 2}=$ 1280.6 at the end of the plate.

To further characterize the state and shape of the boundary layer, we examined the development of the momentum thickness $(\theta)$ and shape factor $(H)$ along the wall (Figure 3 ). The results were found to lie within the envelope of the established theories. Moreover, the high value of the shape factor $(H)$ emphasizes the compressibility effects occurring in highspeed flows [21], while the boundary layer always remains attached to the wall. 
a)

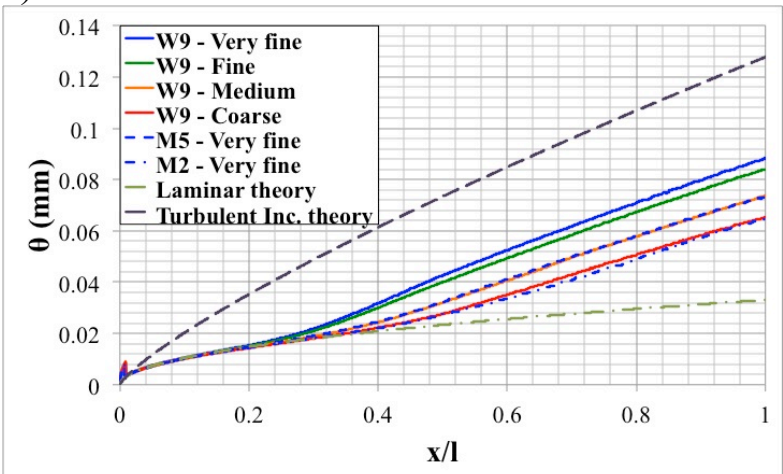

b)

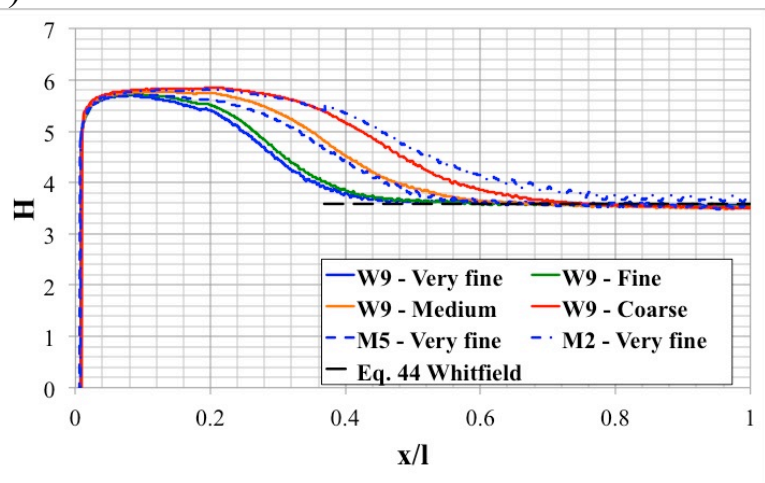

Figure 3: a) Momentum thickness $\theta$ development along the plate. Theoretical models for fully laminar and incompressible fully turbulent flows are also presented. $b$ ) Shape factor $\mathrm{H}$ along the plate.

Furthermore, an investigation of the acoustic behavior of the supersonic boundary layer was performed. The sound pressure levels (SPL) are shown in (Figure 4a). High levels of sound are produced at the leading edge of the plate where a weak shock occurs as the freestream flow intersects with the plate. In the laminar region and downstream of the leading edge the sound levels decrease up to the transition point. At this point, we observe a sudden rise in the SPL culminating just before the flow becomes fully turbulent.

Even though all the numerical schemes considered here similarly predict the peak sound levels, the position of the maxima differs notably. The overshoot of the SPL in the transition region $[22,23]$ emphasizes the criticality of accurately predicting the transition point. In the fully turbulent regime, all schemes converge to a similar SPL.

a)

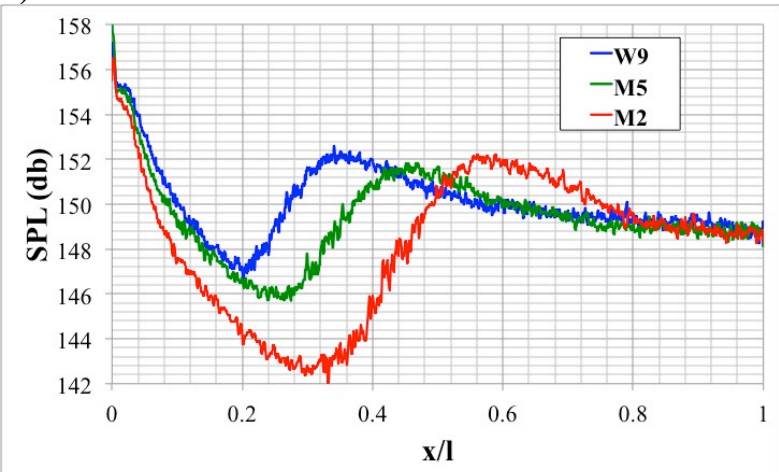

b)

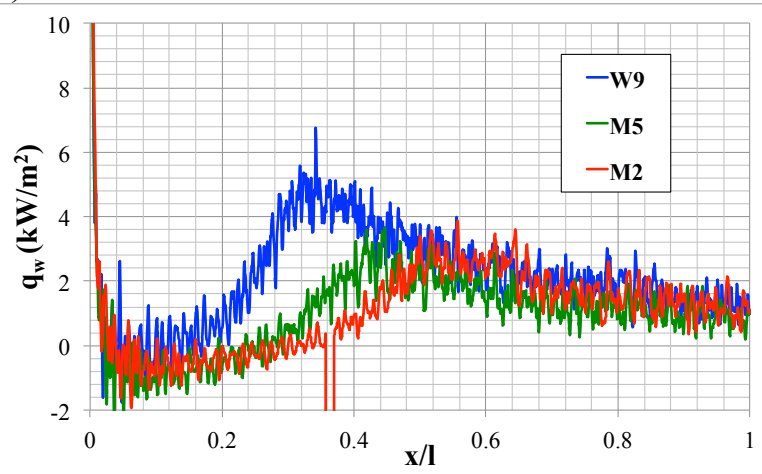

Figure 4: a) SPL of the pressure RMS fluctuations on the wall along the plate. b) Heat transfer $q_{w}$ from the fluid flow to the wall along the plate.

High levels of acoustic loading in the transition region coalesce with high heat loads (Figure $4 \mathbf{b}$ ). The chosen temperature of the isothermal wall is close to the adiabatic temperature, which justifies the nearly zero heat transfer in the laminar region. Heat loads in the turbulent region are higher than those calculated in the laminar one but are nearly one third of the peak value in the transition region. In the transitional regime, results differ considerably with those of the lower-order schemes, which predict lower heat transfer rates. This is attributed to the dissipative behavior of lower-order of numerical accuracy. The dependence of thermal loads on the state of the boundary layer (laminar, transitional, or turbulent), as well as the overshoot in the transitional region, are in agreement with past experimental and numerical results $[24,25,26]$. The heat transfer loads in the fully laminar 
and turbulent regions are influenced slightly by the order of the numerical accuracy; however, the mesh size is more important in this case.

\section{B. Supersonic flow over a compression ramp}

For the second case, we considered a SWTBLI over a compression ramp inclined at an angle of $24^{\circ}$, which was investigated by Wu and Martin [27] using DNS with free-stream conditions of Mach 2.9 and $\mathrm{Re}_{\delta}$ of 38,700. The presence of the inclined surface gives rise to a shock wave, which interacts with the incoming turbulent boundary layer leading to the formation of a separation bubble and a $\lambda$-shockwave. The size of the separation region is dictated by the intensity of the incoming turbulent flow and the strength of the formed shock wave. A sketch illustrating the most important physical processes that take place is given in Figure 5.

Table 3: Simulation parameters for the second case.

\begin{tabular}{cccc}
\hline$\delta_{0}$ & $\mathrm{U}_{\infty}$ & $\mathrm{T}_{\infty}$ & $\mathrm{M}$ \\
$6.4 \mathrm{~mm}$ & $609.1 \mathrm{~m} / \mathrm{s}$ & $107.1 \mathrm{~K}$ & 2.9 \\
\hline \hline$\mu_{\infty}$ & $\rho_{\infty}$ & $\mathrm{T}_{\mathrm{w}} / \mathrm{T}_{\infty}$ & $\mathrm{Re}_{\delta}$ \\
$7.7488 \times 10^{-6} \mathrm{~Pa} \mathrm{~s}$ & $0.077 \mathrm{~kg} / \mathrm{m}^{3}$ & 2.87 & 38737 \\
\hline
\end{tabular}

The physical properties and computational domain are similar to those of previous DNS studies and are provided in Tables 3 and Table 4, respectively. Because the numerical scheme still needs to capture and resolve the synthetic inflow perturbations that lead to a turbulent flow, the length of the upstream domain was increased relative to the DNS by approximately five boundary layer heights $\left(\delta_{0}\right)$. The spanwise length was also increased to investigate possible large-scale structures that may develop in the post-shock region after re-attachment has occurred.

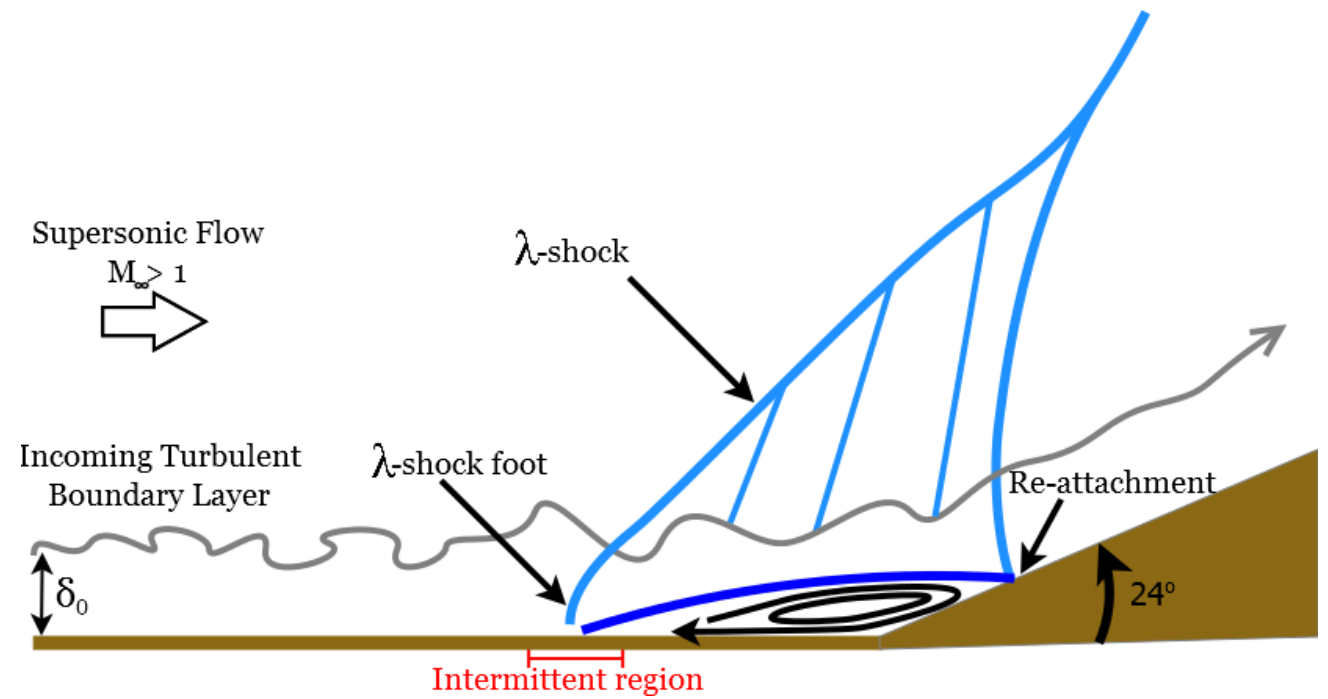

Figure 5: Schematic illustrating the flow over a compression ramp.

Periodic boundary conditions were used in the spanwise $(\mathrm{z})$ direction. In the wall-normal (y) direction, a no-slip isothermal wall $\left(\mathrm{T}_{\mathrm{W}}=309 \mathrm{~K}\right)$ was used. A supersonic outflow condition was imposed at the outlet and upper boundary opposite to the wall. The boundary condition at the inlet required assigning the turbulent boundary layer accurately. A synthetic 
turbulent digital filter approach was further developed in conjunction with the present numerical framework [28] to generate the incoming turbulent boundary layer data. An integral length scale of $0.22 \delta_{0}$ in the streamwise direction was chosen for the digital filter turbulent inflow technique. For the spanwise and wall-normal directions, the integral length scales chosen were $0.2 \delta_{0}$ and $0.5 \delta_{0}$, respectively.

Table 4: Mesh parameters for the second case.

\begin{tabular}{llllllllll}
\hline & $\mathrm{x}_{\max }$ & $\mathrm{y}_{\max }$ & $\mathrm{z}_{\max }$ & $\mathrm{n}_{\mathrm{x}}$ & $\mathrm{n}_{\mathrm{y}}$ & $\mathrm{n}_{\mathrm{z}}$ & $\Delta \mathrm{x}^{+}$ & $\Delta \mathrm{y}^{+}$ & $\Delta \mathrm{z}^{+}$ \\
\hline iLES & 21.4 & 5 & 3 & 1128 & 168 & 120 & 41.51 & 3.5 & 51.92 \\
DNS [29] & 15.4 & 5 & 2.2 & 1024 & 160 & 128 & 31.23 & 0.2 & 28.56 \\
\hline
\end{tabular}

Note that, in this test case, the LM correction was implemented only in conjunction with the MUSCL schemes (M2LM and M5LM).

The streamwise distribution of the temporally and spatially averaged mean wall pressure (Figure 6) shows that the W9 scheme provides more accurate results than the M2LM and M5LM schemes and are in excellent agreement with the DNS [27] and experiment [30] results. The M2LM and M5LM schemes (blue dot-dashed and dashed lines, respectively) are characterized by a delay in the location of the separation bubble. This is due to large-scale structures that are predominant in the flow and persist due to the lack of resolved finer scales to assist in their natural breakdown mechanism. Larger turbulent scales are capable of carrying greater amounts of kinetic energy and momentum from the freestream and transferring them down to the wall. As a result, separation is significantly delayed in the case of the M2LM and M5LM schemes.

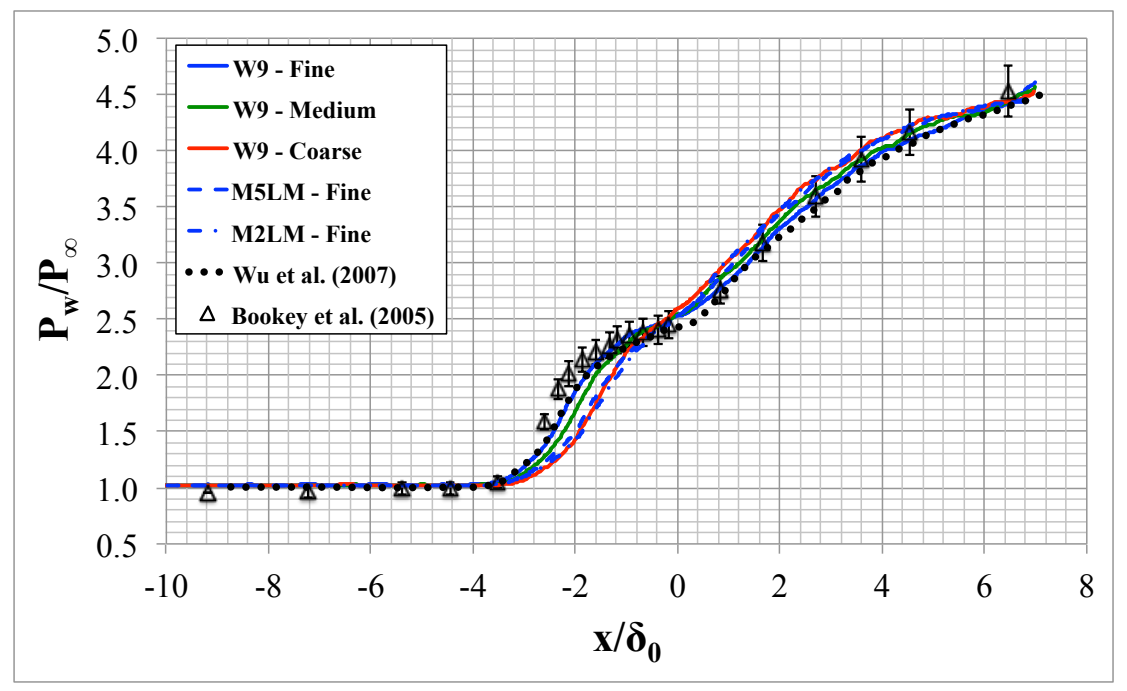

Figure 6: Mean wall pressure distribution in the streamwise direction.

The incoming mean streamwise velocity profile obtained by the digital filter technique at the inflow $\left(\mathrm{x} / \delta_{0}=-15\right)$ is compared to a further two downstream locations, positioned at $\mathrm{x} / \delta_{0}$ $=-11$ and $\mathrm{x} / \delta_{0}=-8$. This allows the observation of the streamwise evolution of the turbulent boundary layer created by the digital filter technique. The position at $\mathrm{x} / \delta_{0}=-8$ is a common location for both DNS and iLES upstream of the compression ramp corner and SWTBLI and therfore the velocity profiles should closely match. Both the M5LM and W9 schemes show very good agreement to the DNS and experiment as seen in Figure 7, however the W9 results are marginally more accurate near to the wall $\left(\mathrm{y} / \delta_{0} \approx 0.1\right)$, as well as near $\mathrm{y} / \delta_{0}=0.5$. Therefore, the synthetic turbulent field created by the digital filter technique requires 
approximately $6 \delta_{0}$ in the streamwise direction to adjust. Note that the DNS study [27] used the rescaling method developed by $\mathrm{Xu}$ and Martin [29] to generate the turbulent inflow condition, with the recycling station located at $4.5 \delta_{0}$ downstream of the inlet.

a)

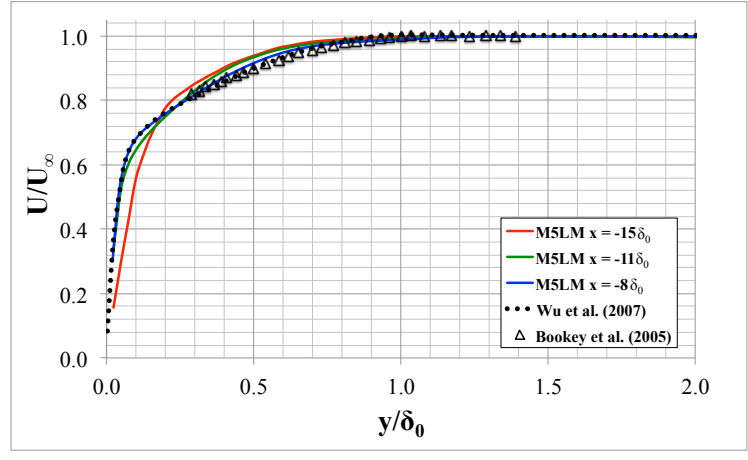

b)

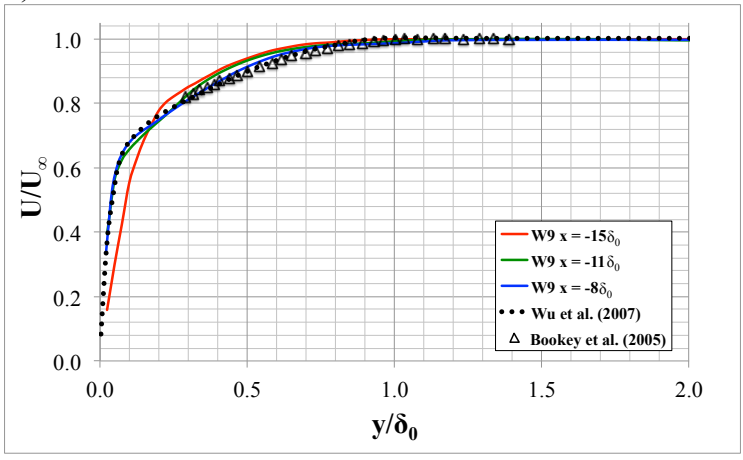

Figure 7: Comparison of inflow streamwise velocity to the DNS and Experiment using the W9 and M5LM schemes on the fine grid.

By the time the flow reaches the intermittent region where the foot of the $\lambda$-shockwave interacts with the separation bubble that formed (as illustrated in Figure 5), there is a considerable difference in the mean streamwise boundary layer profile between the two schemes, as shown in Figure 8a. Because the position of the onset of the separation bubble was not accurately captured using the M2LM and M5LM schemes, as hinted earlier by the mean wall pressure streamwise distribution given in Figure 6, this finding comes as no surprise. Nonetheless, the W9 scheme shows a very good agreement with the DNS in this challenging region of the flow.

a)

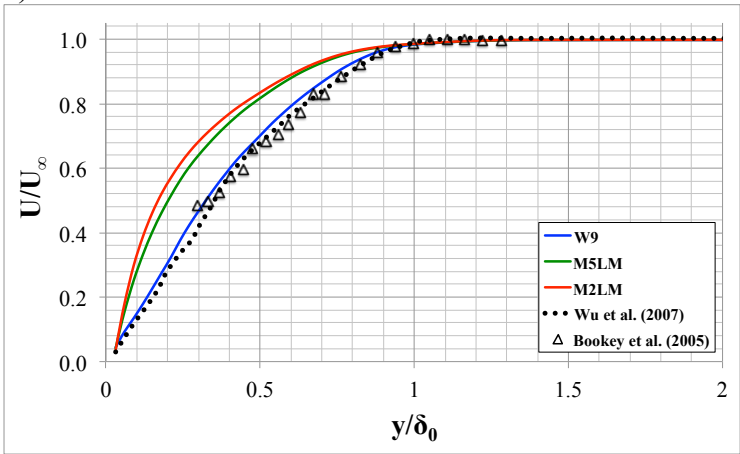

b)

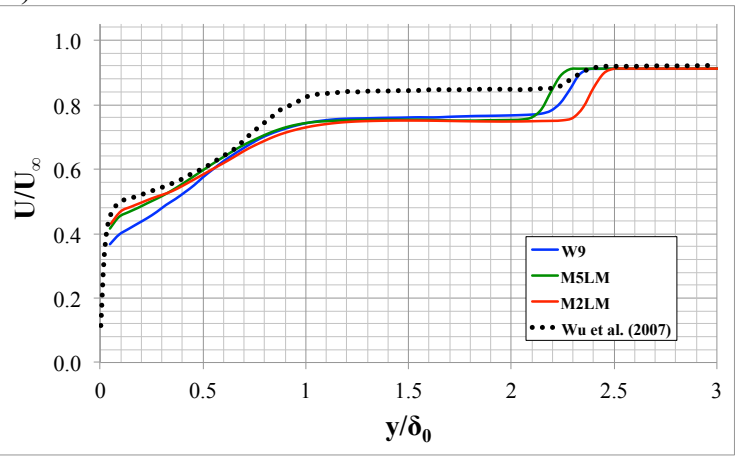

Figure 8: Streamwise velocity profiles at two different streamwise positions, a) $x / \delta_{0}=-1.9$ and $b$ ) $x / \delta_{0}=6.1$.

Near the outlet at $\mathrm{x} / \delta_{0}=6.1$, the velocity profiles of all schemes (M2LM, M5LM, and W9) show a greater deviation from those of the DNS. The freestream velocity tangential to the wall obtains a similar value to that of the DNS down to $y / \delta_{0}=2.5$ for all schemes; However, only the W9 location, where the shockwave passes, is in closer agreement to the DNS. All methods however fail to accurately capture the DNS profile below this point, possibly due to the rapid coarsening of the streamwise grid resolution as the outlet is approached.

Figure 9 shows a qualitative comparison between the M5LM and W9 schemes with respect to the resolved turbulent flow by plotting the isosurfaces of the vortex cores. W9 resolves more vortices than M5LM upstream of the separation region. However, differences between the two schemes become less apparent downstream of the SWTBLI. The M2LM 
scheme is not shown here but it has similar behavior to M5LM and resolves even fewer vortices.

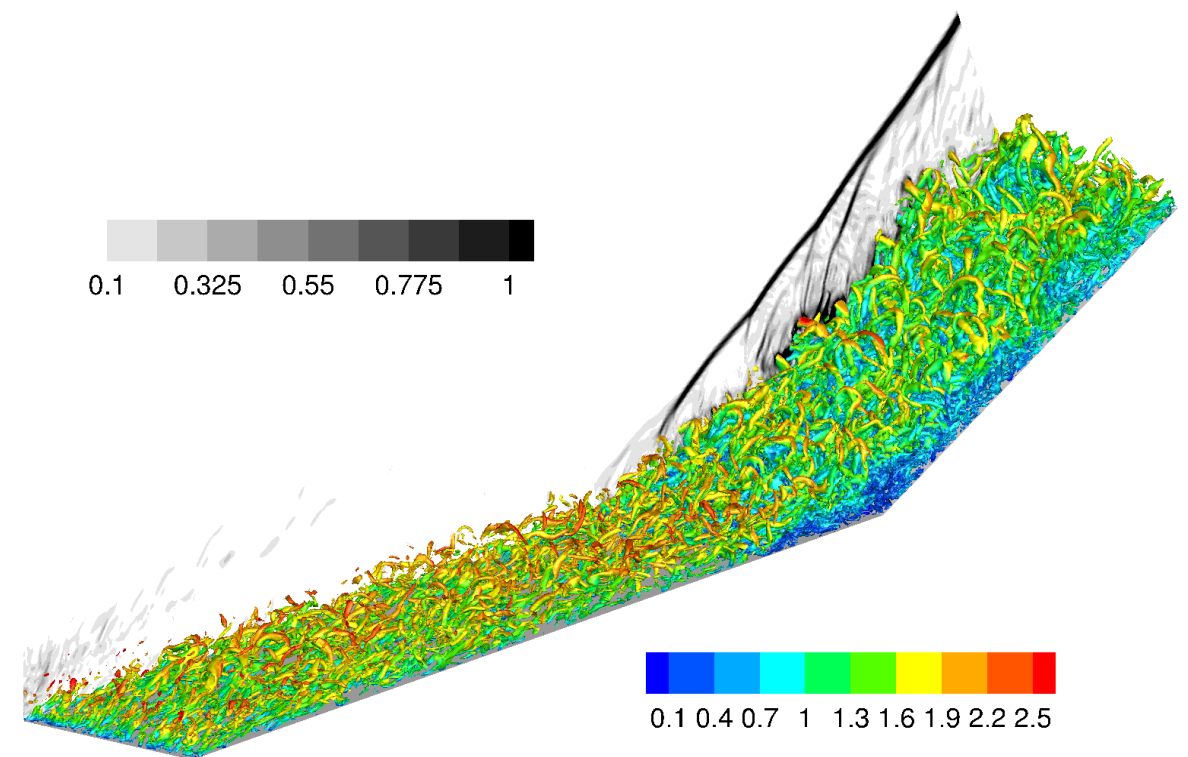

a)

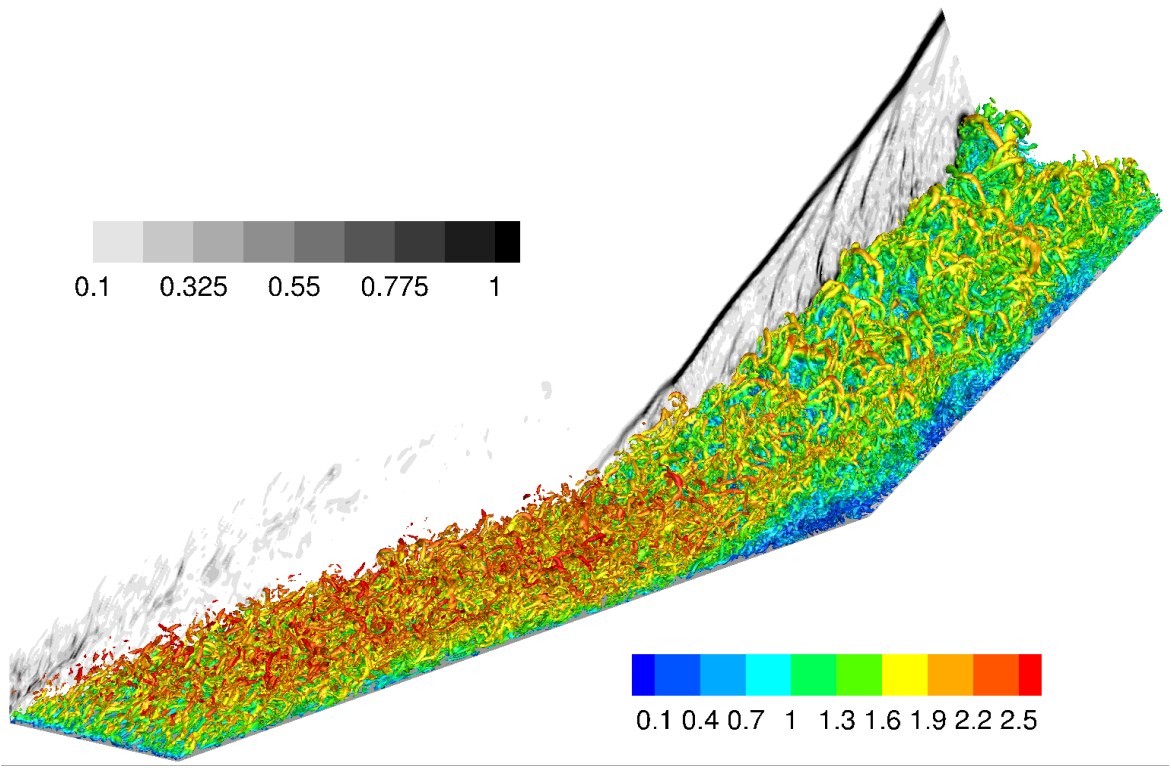

b)

Figure 9: Iso-surfaces of Q-criterion colored by Mach number $\left(=2 / U_{\infty}^{2}\right)$ for a) M5LM and b) W9, respectively; density gradient magnitude $|\nabla \rho| / \rho_{\infty}$ contours plane is in grayscale.

Even though the flow is supersonic in the freestream, the boundary layer still contains a subsonic region. For the considered case, the boundary layer remains subsonic in the first 5$10 \%$ of the incoming boundary layer height, which can contain upward of 20 cells for a relatively fine grid. The subsonic region of the separation bubble, however, can reach a size of $0.6 \delta_{0}$, and any loss of accuracy here could potentially lead to significant errors. However, the coefficient of friction $\left(\mathrm{C}_{\mathrm{f}}\right)$ plotted in Figure 10 suggests that the separation bubble is primarily influenced by the resolved upstream turbulent boundary layer because the 
application of the low Mach number treatment to the fifth-order MUSCL (M5 versus M5LM) scheme hardly shows any effect. By contrast, there is a slight but noticeable improvement in $\mathrm{C}_{\mathrm{f}}$, particularly in the evolving turbulent boundary layer region upstream of the separation bubble, suggesting an increase in the resolved wall velocity. Interestingly, this does not translate to an improvement, or in fact a difference, in $\mathrm{C}_{\mathrm{f}}$, which might indicate that the onset and behavior of the separation bubble is primarily dominated by the (resolved) turbulent dynamics in the supersonic region of the boundary layer.

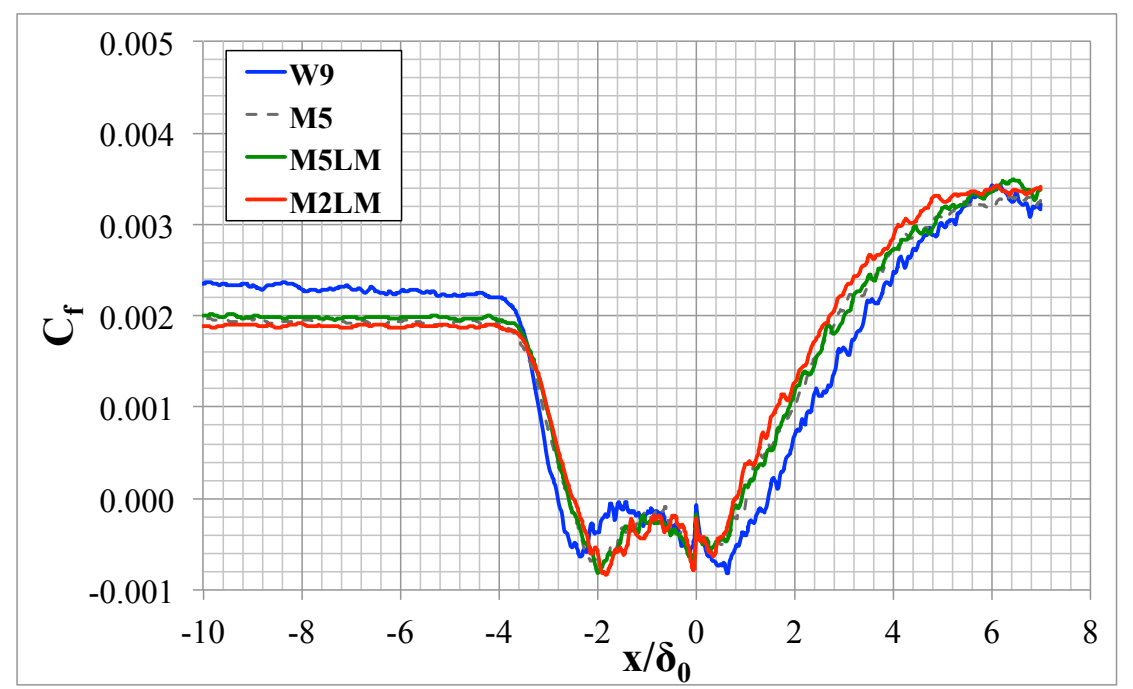

Figure 10: Coefficient of friction distribution.

Finally, as in the first case, we performed an investigation of the acoustic behavior of the SWTBLI. The SPLs are shown in Figure 11a. At the region of the $\lambda$-shock foot (the initial part of the separation bubble), we observe a significant increase in the SPLs. After the separation bubble, the SPLs continue to increase with a lower rate reaching a peak at $\mathrm{x} / \delta_{0} \sim 3$. The SPLs decline after this point but are still high enough in order to expose the panel structure to significant acoustic fatigue. The lower-order schemes overpredict the magnitude of the SPL at the critical region of the separation bubble and in the upstream turbulent boundary layer. In addition, the positions of the SPL jump and the maximum value are not accurately predicted with M5LM and M2LM.

a)

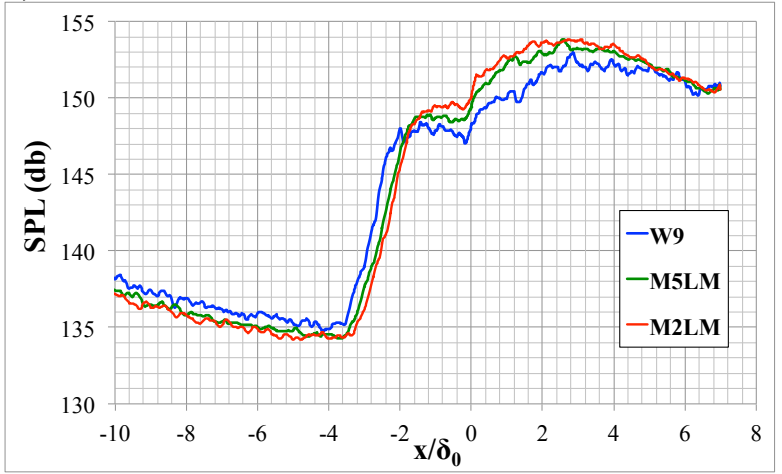

b)

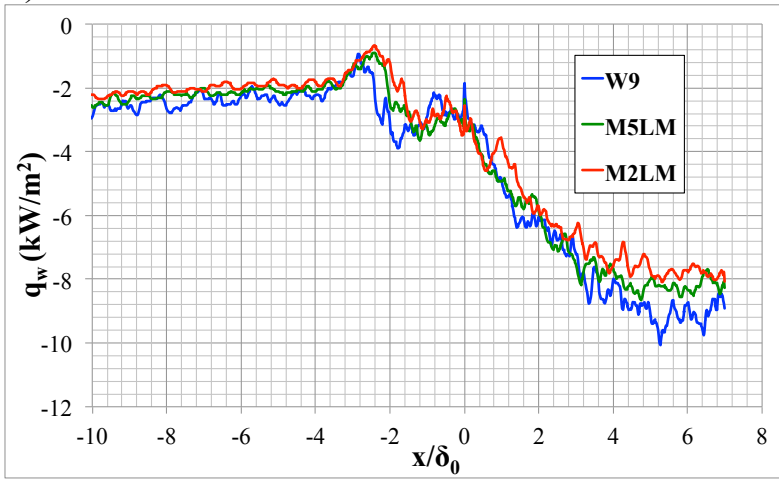

Figure 11: a) SPL of the pressure RMS fluctuations on the wall along the ramp. b) Heat transfer $q_{w}$ from the fluid flow to the wall along the ramp.

The temperature of the wall was kept constant during the simulation at a value slightly higher than the adiabatic recovery temperature. For that reason, and contrary to the first case, heat is transferred from the wall to the fluid (Figure 11b). In the initial part of the TBL, and 
before the separation bubble, the heat transfer is nearly constant. At the intermittency area the fluid close to the wall is suddenly heated and the heat transfer rate decreases. Inside the bubble separation region the heat transfer rate is nearly stabilized but at a higher rate than that of the upstream TBL region. In the downstream, the TBL heat transfer rate keeps steadily increasing until a plateau is reached close to the end of the simulation domain. The fluctuations of the heat transfer rate along the inclined surface are significant and should be carefully considered during the design process of supersonic and hypersonic planes. The heat transfer loads along the ramp are not greatly influenced by the order of the numerical accuracy.

\section{Concluding remarks}

The acoustic and thermal behavior of transitional and turbulent boundary layers in highspeed flows was investigated while taking into account the effect of the numerical accuracy of the different schemes employed.

The thermoacoustic loading is higher in the transitional regime. The order of the numerical scheme has a large effect on the predictions made in the transitional flow region, whereas the results obtained by the different schemes converge in the laminar and fully turbulent flow regions.

LM correction was employed in the SWTBLI case but was found to provide little to no improvement in the accuracy of the results. For schemes such as the ninth-order WENO, where numerical dispersion becomes large and numerical dissipation diminishes, the low Mach number treatment was found to deteriorate the accuracy of the results. Overall, the ninth-order WENO was found to give very promising results in iLES for transitional highspeed flows over a flat plate as well as SWTBLI over a compression ramp.

\section{Acknowledgements}

This work was sponsored by the Air Force Office of Scientific Research, Air

Force Material Command, USAF, under grant number FA9550-14-1-0224. The U.S. Government is authorized to reproduce and distribute reprints for Governmental purpose notwithstanding any copyright notation thereon. Results were obtained using the EPSRC funded ARCHIE-WeSt High Performance Computer (www.archie-west.ac.uk) under EPSRC grant no. EP/K000586/1.

\section{References}

[1] M. Bernardini and S. Pirozzoli, "Wall pressure fluctuations beneath supersonic turbulent boundary layers," Physics of Fluids, vol. 23, no. 8, 2011.

[2] L. Duan, M. M. Choudhari and M. Wu, "Numerical Study of Pressure Fluctuations due to High-Speed Turbulent Boundary Layers," in 42nd AIAA Fluid Dynamics Conference and Exhibit, New Orleans, 2012.

[3] C. Ostoich, D. Bodony and P. Geubelle, "Interaction of a Mach 2.25 turbulent boundary layer with a fluttering panel using direct numerical simulation," Physics of Fluids, vol. 25, no. 11, 2013.

[4] J. Boris, F. Grinstein, E. Oran and R. Kolbe, "New insights into large eddy simulation," Fluid Dynamics Research, vol. 10, no. 4-6, pp. 199-228, 1992.

[5] C. Fureby and F. F. Grinstein, "Large Eddy Simulation of High-Reynolds-Number Free and Wall-Bounded Flows," Journal of Computational Physics, vol. 181, no. 1, pp. 68-97, 2002.

[6] L. G. Margolin, W. J. Rider and F. F. Grinstein, "Modelin turbulent flow with implicit LES," Journal of Turbulence, vol. 7, no. 15, 2006.

[7] I. W. Kokkinakis and D. Drikakis, "Implicit Large Eddy Simulation of weakly-compressible turbulent 
channel flow," Computer Methods in Applied Mechanics and Engineering, vol. 287, pp. 229-261, 2015.

[8] J. Poggie, N. J. Bisek and R. Gosse, "Resolution effects in compressible, turbulent boundary layer simulations," Computers and Fluids, vol. 120, pp. 57-69, 2015.

[9] E. F. Toro, Riemann Solvers and Numerical Methods for Fluid Dynamics, 3rd ed., Springer, 2009.

[10] B. van Leer, "Towards the ultimate conservative difference scheme III. Upstream-centered finite-difference schemes for ideal compressible flow," Journal of Computational Physics, vol. 23, no. 3, pp. 263-275, 1977.

[11] K. H. Kim and C. Kim, "Accurate, efficient and monotonic numerical methods for multidimensional compressible flows: Part II: Multidimensional limiting process," Journal of Computational Physics, vol. 208, no. 2, pp. 570-615, 2005.

[12] D. S. Shu and C.-W. Balsara, "Monotonicity preserving weighted essentially non-oscillatory schemes with increasingly high order of accuracy," Journal of Computational Physics, vol. 160, no. 2, pp. 405-452, 2000.

[13] B. Thornber, A. Mosedale, D. Drikakis, D. Youngs and R. J. R. Williams, "An Improved Reconstruction Method for Compressible Flows with Low Much Number Features," Joutnal of Computational Physics, vol. 227, pp. 4873-4894, 2008.

[14] R. Spiteri and S. J. Ruuth, "New Class of Optimal High-Order Strong-Stability-Preserving Time Discretization Methods," SIAM Journal on Numerical Analysis, vol. 40, no. 2, pp. 469-491, 2002.

[15] P. Tsoutsanis, I. W. Kokkinakis, L. Konozsy, D. Drikakis, R. J. R. Williams and D. Youngs, "Comparison of structured and unstructured-grid, compressible and incompressible methods using the vortex pairing problem," Computer Methods in Applied Mechanics and Enginnering, vol. 293, pp. 207-231, 2015.

[16] H. Schlichting, Boundary-Layer Theory, McGraw-Hill Book Company, 1979.

[17] F. M. White, Fluid Mechanics, McGraw-Hill, 2011.

[18] S. Pirozzoli and M. Bernardini, "Turbulence in supersonic boundary layers at moderate Reynolds number," Journal of Fluid Mechanics, vol. 688, pp. 120-168, 2011.

[19] M. Eléna and J. P. Lacharme, "Experimental study of a supersonic turbulent boundary layer using a laser Doppler anemometer," Journal de Méchanique Théorique et Appliquée, vol. 7, no. 2, pp. 90-175, 1988.

[20] J. M. Österlund, A. V. Johansson, H. M. Ngib and M. H. Hites, "A note on the overlap region in turbulent boundary layers," Physics of Fluids, vol. 12, no. 1, pp. 1-4, 2000.

[21] D. L. Whitfield, "Integral Solution of Compressible Turbulent Boundary Layers Using Improved Velocity Profiles," 1978.

[22] K. Casper, S. Beresh, J. Henfling, R. Spillers, B. Pruett and S. Schneider, "Hypersonic Wind-Tunnel Measurements of Boundary-Layer Pressure Fluctuations," AIAA Paper 2009-4054, 2009.

[23] K. Casper, S. Beresh, J. Henfling, R. Spillers and B. Pruett, "High-Speed Schlieren Imaging of Disturbances in a Transitional Hypersonic Boundary Layer," AIAA Paper 2013-0376, 2013.

[24] R. Narasimha, "The Laminar-Turbulent Transition Zone in the Boundary Layer," Progress is Aerospace Sciences, vol. 22, no. 1, pp. 29-80, 1985.

[25] T. P. Wadhams, E. Mundy, M. MacLean and M. Holden, "Ground Test Studies of the HIFiRE-1 Transition Experiment Part 1: Experimental Results," Journal of Spacecraft and Rockets, vol. 45, no. 6, pp. 11341148, 2008.

[26] K. Franko and S. Lele, "Breakdown Mechanisms and Heat Transfer Overshoot in Hypersonic Zero Pressure Gradient Boundary Layers," Journal of Fluid Mechanics, vol. 730, pp. 491-532, Aug. 2013.

[27] M. Wu and . M. P. Martin, "Direct Numerical Simulation of Supersonic Turbulent Boundary Layer over a Compression Ramp," AIAA Journal, vol. 45, no. 4, pp. 879-889, 2007.

[28] Z. A. Rana, B. Thornber and D. Drikakis, "Transverse jet injection into a supersonic turbulent cross-flow," Physics of Fluids, vol. 23, no. 4, 2011.

[29] S. Xu and M. P. Martin, "Assessment of Inflow Boundary Conditions for Compressible Turbulent Boundary Layers," Physics of Fluids, vol. 16, no. 7, pp. 2623-2639, 2004.

[30] P. B. Bookey, C. Wyckham, A. J. Smits and M. P. Martin, "New Experimental Data of STBLI at DNS/LES Accessible Reynolds Numbers," in 43rd AIAA Aerospace Sciences Meeting and Exhibit, Reno, 2005.

[31] D. Coles, "The law of the wake in the turulent boundary layer," Journal of Fluid Mechanics, vol. 1, pp. 191-226, 1956.

[32] J. M. Österlund, A. V. Johansson, H. M. Nagib and M. H. Hites, "A note on the overlap region in turbulent boundary layers," Physics of fluids, vol. 12, pp. 1-4, 2000.

[33] J. Kim, P. Moin and R. Moser, "Turbulence statistics in fully developed channel flow at low Reynolds 
number," Journal of Fluid Mechanics, vol. 177, pp. 133-166, 1987. 